\title{
La evaluación de la integración de las TIC en la educación superior: fundamento para una metodología
}

\section{The Evaluating of Integration of ICT in Higher Education: Foundation for a Methodology}

\author{
Pedro Nolasco Vázquez \\ Universidad Veracruzana. México. \\ pnolascov@gmail.com \\ Mario Miguel Ojeda Ramírez \\ Universidad Veracruzana. México. \\ mojeda@uv.mx
}

\begin{abstract}
Resumen
Se sustenta y propone una metodología que permite el estudio de la incorporación de las tecnologías de la información y comunicaciones (TIC) en la educación superior, desde el enfoque del comportamiento organizacional post-implementación tecnológica. Convencionalmente, las metodologías propuestas para este fin están basadas en el Capability Maturity Model (CMM): a mayor madurez digital de los procesos clave, mayor madurez organizacional (Duarte \& Ventura, 2011). Sin embargo, el CMM no considera la relación de los individuos en la organización con la tecnología implementada, lo que puede influir en diagnósticos incompletos, la rentabilidad de la inversión y el éxito de la implementación. Por tal motivo, se propone una metodología fundamentada en el comportamiento organizacional post-implementación tecnológica, que se complementa con el concepto de apropiación tecnológica y el modelo conceptual para la medición de las TIC en la educación de la UNESCO (2009). La prueba piloto permitió evaluar su viabilidad para la elaboración de diagnósticos preliminares.
\end{abstract}

\section{Palabras clave}

Indicadores TIC en educación superior; Aceptación tecnológica; Rutinización tecnológica; Infusión tecnológica

\begin{abstract}
A methodology to study the incorporation of information technology and communications (ICT) in higher education is supported and proposed, from the perspective of organizational behavior post-implementation technology. Conventionally, the proposed methodologies for this purpose are based on the Capability Maturity Model (CMM): the higher the digital maturity of key processes, greater organizational maturity (Duarte \& Ventura, 2011). However, the CMM does not consider the relationship of individuals in the organization with the implemented technology, which may influence incomplete diagnoses, return on investment and successful implementation. Therefore, a methodology based on the postimplementation organizational behavior technology, complemented with the concept of technological appropriation and the conceptual model for the measurement of ICT in education UNESCO (2009) is proposed. The pilot test was used to assess the feasibility of the model for the development of preliminary diagnoses.
\end{abstract}

\section{Key words}

ICT Indicators in higher education; Technological acepptance; Technological routinization; Technological infusion 


\section{Introducción:}

La medición de la incorporación tecnológica en las organizaciones ha sido abordada desde los modelos de madurez de la ingeniería de procesos, principalmente a partir del Capability Maturity Model (CMM) (Duarte \& Ventura, 2011). Este modelo plantea una ruta hacia el mejoramiento de procesos de software (Inicial, Repetible, Definido, Administrado, Optimizado), donde a mayor nivel de madurez, mayor calidad de servicios a clientes (Curtis, Hefley \& Miller, 1995; Paulk, Curtis, Chrissis \& Webber, 1993). La aplicación de este modelo ha sido extendida a las universidades (Duarte \& Ventura, 2011; López, 2015). Otra vertiente para la medición de la incorporación tecnológica universitaria es la del Gobierno de Tecnologías de Información (IT Government) -basada también en el CMM- promovido por el estándar COBIT del IT Governance Institute (2007), que plantea la interacción de las gerencias administrativa y de tecnologías de información para lograr, entre otras cosas, la alineación de la tecnología con los objetivos de negocio de la organización (Fernández \& Llorens, 2011).

Sin embargo, las propuestas basadas en el CMM no consideran el aspecto humano; es decir, no considera la relación de las personas con la tecnología, lo cual puede incidir en diagnósticos incompletos, además de representar un obstáculo en la implementación de proyectos tecnológicos (Saga \& Zmud, 1994; Quijano, 2007; Zubieta, Bautista \& Quijano, 2011). Esta problemática ha sido abordada desde el comportamiento organizacional post-implementación de Saga y Zmud (1994) y desde la apropiación tecnológica (Cobo, 2008; Crovi, 2009; Van Dijk, 2005; Colás \& Rodríguez, 2005; Overdijk \& Van Diggelen, 2006; Pimienta, 2007; INFOTEC, 2014). La propuesta de evaluar la relación de los individuos con la tecnología como indicador de la integración tecnológica tiene sentido porque se entiende que en la medida en que esta relación aumenta, en esa medida se cuenta con un sistema de información que se identifica con las tareas cotidianas, estratégicas y de toma de decisiones en la organización: a mayor nivel de relación humano-tecnológica, mayor incrustación de la implementación tecnológica, de manera profunda y comprensiva, en los sistemas de trabajo de una organización o individuo (Sullivan, 1985; Kwon, 1987; Cooper \& Zmud, 1990).

Saga y Zmud (1994), en el marco de su modelo de comportamiento postimplementación de TIC en una organización, refieren a la "Aceptación", "Rutinización" e "Infusión" tecnológicas como niveles progresivos de incorporación tecnológica, que hacen alusión respectivamente al uso de la tecnología de manera voluntaria; a la institucionalización de la tecnología; y a la incrustación tecnológica en los sistemas de trabajo de la organización para la potenciación de los objetivos organizacionales. Por su parte, respecto de la apropiación tecnológica, Van Dijk (2005) expresa que es consecuencia de la motivación, acceso y desarrollo de habilidades digitales; Crovi (2009) la presenta como la integración de las TIC a la práctica social en un ámbito histórico específico; Pimienta (2007) expone que es un proceso de aprendizaje que permite que las personas, grupos u organizaciones tengan control sobre el uso tecnológico en coherencia con su entorno.

En esta línea de trabajo, se cuenta con investigaciones basadas en la aceptación tecnológica, como el desarrollado con docentes de la UNAM (Zubieta et al., 2011) y el de cambio organizacional en una biblioteca académica (Quijano, 2007), así como las basadas en la apropiación tecnológica como el estudio sobre estudiantes de diferentes

La evaluación de la integración de las TIC en la educación superior: fundamento para una metodología. 
áreas disciplinarias de la Universidad Veracruzana (Álvarez, 2015) y el de diferentes modalidades educativas en universidades de México, Guatemala y Venezuela (Fernández, 2015).

Por otro lado, la UNESCO (2009) introduce los conceptos de "e-aptitud digital" (disposición y acceso), "e- intensidad" (desarrollo y uso de contenidos digitales) y "eimpacto" (efecto) para responder a la emergencia del monitoreo del uso de las TIC en la educación de los países desde una perspectiva internacional. Esta propuesta ha sido aplicada en el estudio de la aptitud digital en países de América Latina y el Caribe (UNESCO, 2013). Igualmente, se dan cuenta de estudios con similitud metodológica para medir la incorporación digital en el orbe (World Wide Web Foundation [WWWF], 2012; 2013; 2014; World Economic Forum [WEF], 2015; Unión Internacional de Telecomunicaciones [UIT], 2014). En México, se aprecia la aproximación metodológica en la Agenda Digital del Gobierno Federal de México (SCT, 2012), que propone el uso de la tecnología con fines de equidad social y competitividad, bajo las vertientes progresivas de conectividad, contenidos y apropiamiento.

Si las propuestas basadas en el CMM aplicadas a la universidad no consideran el aspecto de la relación humana con la tecnología, se ignora un componente relevante que favorece el éxito de la implementación tecnológica (Kwon \& Zmud, 1987; Cooper \& Zmud, 1990) y que puede ser crucial para detonar el potencial de la inversión tecnológica (Sullivan, 1985; Zmud \& Apple, 1992).

Este artículo propone el estudio de la integración tecnológica universitaria desde la aceptación, rutinización e infusión tecnológicas de Saga y Zmud (1994); y de la adopción del concepto de la apropiación tecnológica (Davis, 1986; Crovi, 2009; Van Dijk, 2005; Colás \& Rodríguez, 2005; Pimienta, 2007; INFOTEC, 2014) y el marco conceptual para el desarrollo de indicadores TIC en la educación de la UNESCO (2009). Se pretende que la metodología propuesta pueda contribuir al diagnóstico efectivo, rentabilizar la inversión financiera, la mejora continua y al diseño de política digital universitaria.

Para sustentar la propuesta se realiza una revisión de los conceptos y aportaciones teóricas; posteriormente se desarrolla la propuesta metodológica. Se hace una valoración de la factibilidad de la implementación y se comentan las experiencias de una instrumentación piloto.

\section{El marco referencial:}

\section{1.- El comportamiento organizacional post-implementación}

En el marco del modelo de comportamiento post-implementación tecnológica en las organizaciones de Saga y Zmud (1994) se considera a la "Aceptación", la "Rutinización" e "Infusión" tecnológicas como niveles progresivos de incorporación tecnológica,

\section{La aceptación tecnológica}

Los conceptos fundamentales son las "creencias", "actitudes", "intenciones" y "comportamiento". Las primeras, aluden a la dimensión de certidumbre asociada a un 
atributo específico de algún objeto (Davis, 1986); las segundas, refieren a la dimensión evaluativa en relación a algún objeto con tendencia de estabilidad sobre el tiempo y resulta del entrenamiento o experiencias pasadas (Nunally, 1967; Snider \& Osgood, 1969); las terceras, se ubican como una dimensión de probabilidad de una acción relacionada con el objeto; el "comportamiento" refiere a acciones públicas y por tanto observables. La aceptación tecnológica se describe como el acto de recibir el uso de la tecnología voluntariamente y está explicada a partir de las "actitudes", "intenciones" y "frecuencia de uso" además de relaciones adicionales. Por ejemplo, la "intención de uso" por parte de un individuo explica también el compromiso del usuario hacia una tecnología (Kwon, 1987; Cooper \& Zmud, 1990) y refleja la predilección del individuo para engranarse en un comportamiento específico para la "frecuencia de uso". Esta relación está matizada por otras variables adicionales como "conocimiento del usuario": un individuo que posee habilidades y conocimientos modula la aplicación efectiva de una tecnología (Sheppard, Hartwick \& Warshaw, 1988; Davis, 1989).

\section{La rutinización tecnológica}

El término "rutina" hace alusión al hecho de que el uso de la tecnología refleja los procedimientos, hábitos y costumbres de la cotidianidad de la organización. Con el tiempo la implementación tecnológica se llega a considerar un elemento estándar de la rutina de la organización, es decir, la innovación tecnológica se institucionaliza (Zucker, 1977; Ritti \& Silver, 1986). La rutinización no implica que la tecnología implementada sea la más apropiada o que no pueda ser reemplazada posteriormente. Yin (1979) asocia la rutinización a pasajes o ciclos: los pasajes ocurren cuando la innovación tecnológica provoca la transición de un estado de la organización a otro; los ciclos, cuando la innovación persiste a eventos organizacionales periódicos. Así, la rutinización se describe como el logro de pasajes y ciclos que implican ajustes permanentes en el sistema de gobernabilidad de la organización con la presencia de la innovación. A mayor número de pasajes y ciclos mayor rutinización tecnológica. El constructo de la rutinización está determinado por tres variables: "uso percibido como normal" -que implica la institucionalización tecnológica-, "uso estandarizado" -que involucra el uso mediante prácticas y procedimientos consensuados- y "desarrollo de infraestructura administrativa" -que alude al esfuerzo gerencial de apoyo a la implementación-. El modelo está ligado al de la aceptación tecnológica mediante las variables "frecuencia de uso" e "intervención gerencial" e involucra relaciones adicionales. Por ejemplo, la "frecuencia de uso" influye y es influida por las variables "desarrollo de infraestructura administrativa" y "uso percibido como normal". Esta situación puede observarse cuando se establecen políticas o reglas formales asociadas al uso de la aplicación y se favorece el establecimiento de puestos laborales o líneas presupuestarias ligadas a la aplicación tecnológica (como capacitación, soporte o su uso).

\section{La infusión tecnológica}

Las aplicaciones tecnológicas exitosas con el tiempo son mejoradas o reconfiguradas de acuerdo a los cambios de la organización (Kling \& Lacono, 1984). Esto refleja el entendimiento organizacional progresivo del sistema de trabajo y del potencial tecnológico que lo sustenta. En este contexto se entiende el logro de niveles sofisticados de uso tecnológico que potencia el sistema de trabajo. Para optimizar la inversión tecnológica de la organización es necesario realizar la reingeniería de los procesos de trabajo claves para incrementar la capacidad tecnológica (Davenport \& Short, 1990;

La evaluación de la integración de las TIC en la educación superior: fundamento para una metodología. 
Hammer, 1990; King, 1991). Así, el término "infusión” se entiende como el proceso de incrustación de una aplicación tecnológica de manera profunda y comprensiva en los sistemas de trabajo de una organización o individuo (Sullivan, 1985; Kwon, 1987; Cooper \& Zmud, 1990). Zmud y Apple (1992) argumentan que es posible identificar configuraciones de sistemas de trabajo que representen niveles de uso de una tecnología y podría ocurrir en los niveles: (1) "emergente", cuando se usa la tecnología para realizar tareas laborales que no fueron factibles o reconocidas en las fases previas a la aplicación de la tecnología; (2) "integrado", cuando se usa la tecnología para establecer o mejorar conexiones de flujos de trabajo entre un conjunto de tareas laborales; y (3) "extendido", cuando se usan las características de la tecnología para adaptar un conjunto más comprensivo de tareas laborales. El modelo de la infusión tecnológica está ligado a los de la aceptación y rutinización mediante las variables: "intervención gerencial", "actitudes hacia el uso", "conocimiento del usuario", "frecuencia de uso" y "uso estandarizado". Los procesos de infusión, por ejemplo, ejercen efectos fuertes sobre el "conocimiento del usuario" -lo influyen el "uso emergente", "uso integrado", "uso extendido" y "uso estandarizado"-.

\section{2.- La apropiación tecnológica}

Para Cobo (2008) la apropiación tecnológica en la sociedad del conocimiento requiere de tres fases: acceso -conexión tecnológica-, capacitación -habilidades informáticas y acceso a contenidos- y apropiación -colaboración orientada al aprendizaje y generación de nuevo conocimiento-. Crovi (2009) propone igualmente tres fases: acceso, uso y apropiación, entendida la última como la integración de las TIC a la práctica social en un ámbito socio histórico específico. Van Dijk (2005) propone cuatro fases: motivación -como detonante del interés-, acceso, desarrollo de habilidades más allá de lo digital informacionales, instrumentales y de estrategia- y uso -como equivalente de la apropiación. Colás y Rodríguez (2005) exponen que es la forma en que las tecnologías son asumidas por los sujetos influyendo en la interpretación de la realidad y el aprendizaje. Overdijk y Van Diggelen (2006) abundan que es un proceso de construcción social donde las acciones y pensamientos del usuario son impactados por la tecnología. Pimienta (2007) la expone como un proceso de aprendizaje de personas, grupos u organizaciones sobre el uso de las TIC en coherencia con sus entornos. Por su parte, INFOTEC (2014) alude a un proceso de adopción y adaptación tecnológica por parte de un usuario o grupo para incorporarlas en su vida, prácticas y rutinas de trabajo. Puede significar la utilización voluntaria a partir de una necesidad y al mismo tiempo una garantía para la sostenibilidad de proyectos tecnológicos.

\section{3.- Medición de las TIC en la educación según la UNESCO}

La relevancia de monitorear el uso de las TIC en la educación desde una perspectiva internacional implicó consensuar un marco conceptual para el desarrollo de indicadores con viabilidad operacional. Se parte del supuesto de que las necesidades de información para evaluar la incorporación de las TIC en los sistemas educativos evolucionan según se avanza en la implementación de políticas nacionales y según el grado de penetración en los sistemas educativos. En la medida que las TIC se infiltran progresivamente en los sistemas educativos, los indicadores utilizados para monitorear el avance de la política digital evolucionan. Así, se propuso los siguientes niveles progresivos de incorporación de las TIC al sistema educativo: 
- la "e-aptitud digital", se caracteriza por docentes certificados y enseñanza asistida por TIC. Requiere información sobre acceso y uso de infraestructura TIC. Dada, citado en UNESCO (2011) la define como la disposición de la entidad educativa para beneficiarse de las TIC.

- la "e-intensidad", se caracteriza por universidades y bibliotecas virtuales. Requiere información sobre desarrollo de contenidos digitales y gestión de enseñanza innovadora mediada por TIC y autoaprendizaje.

- el "e-impacto", se caracteriza por el uso herramientas como webcasting, podcasting y videoconferencias. Requiere información sobre desempeño académico y laboral, productividad económica y aprendizaje permanente.

Bajo este marco de trabajo, se definieron dominios conceptuales para el diseño de indicadores que monitorean el avance de la incorporación de las TIC en la educación. El proceso de definición se muestra en la Tabla 1: a partir de los niveles de incorporación de las TIC, se desglosa un diseño general de dominios del tipo Entrada-Proceso-Salida (E-P-S). Esto da lugar a un diseño de nivel de detalle intermedio (Diseño específico) que permite la definición de los dominios conceptuales de cada nivel.

Tabla 1. Modelo conceptual para el diseño de indicadores para medir las TIC en la educación (UNESCO, 2009)

\begin{tabular}{|c|c|c|c|}
\hline \multicolumn{4}{|c|}{ Marco conceptual para el diseño de indicadores TIC en la educación } \\
\hline Niveles de incorporación & $\begin{array}{l}\text { Diseño } \\
\text { general } \\
\text { (E-P-S) }\end{array}$ & Diseño específico & $\begin{array}{l}\text { Dominios conceptuales para el } \\
\text { desarrollo de indicadores }\end{array}$ \\
\hline \multirow{4}{*}{$\begin{array}{c}\text { e }- \text { aptitud } \\
\text { Disposición de la entidad } \\
\text { educativa para beneficiarse de } \\
\text { las TIC }\end{array}$} & \multirow{2}{*}{$\begin{array}{l}\text { Políticas } \\
\text { /estrategias }\end{array}$} & \multirow{2}{*}{$\begin{array}{l}\text { Políticas, metas e } \\
\text { incentivos }\end{array}$} & Compromiso político \\
\hline & & & Asociación público-privada \\
\hline & \multirow[b]{2}{*}{ Insumos } & Instalaciones TIC & Infraestructura \\
\hline & & $\begin{array}{l}\text { Certificación de } \\
\text { docentes }\end{array}$ & Desarrollo del personal docente \\
\hline \multirow{2}{*}{$\begin{array}{c}\text { e - intensidad } \\
\text { Desarrollo de contenidos } \\
\text { digitales y gestión de } \\
\text { enseñanza innovadora y } \\
\text { autoaprendizaje }\end{array}$} & \multirow[b]{2}{*}{ Procesos } & Desarrollo curricular & \multirow[b]{2}{*}{$\begin{array}{c}\text { Uso de TIC } \\
\text { (en la enseñanza aprendizaje } \\
\text { curricular) }\end{array}$} \\
\hline & & $\begin{array}{l}\text { Uso de TIC en la } \\
\text { enseñanza aprendizaje }\end{array}$ & \\
\hline \multirow[b]{2}{*}{ e-impacto } & \multirow{4}{*}{$\begin{array}{l}\text { Rendimiento / } \\
\text { resultados }\end{array}$} & \multirow{4}{*}{$\begin{array}{c}\text { Evaluación de } \\
\text { desempeño estudiantil }\end{array}$} & $\begin{array}{l}\text { Participación, competencias y } \\
\text { rendimiento } \\
\end{array}$ \\
\hline & & & \\
\hline $\begin{array}{l}\text { Efectos en el desempeño } \\
\text { académico y laboral, } \\
\text { productividad económica y } \\
\text { aprendizaje permanente }\end{array}$ & & & Resultados e impacto \\
\hline & & & Equidad \\
\hline
\end{tabular}

Los dominios para la "e-aptitud" son: el "Compromiso político" y la "Asociación público-privada" que refieren a información sobre la disponibilidad de políticas, programas o marcos normativos que definan la implementación de las TIC en la educación; "Infraestructura" que sugiere información respecto de la cantidad y calidad de instalaciones o recursos relacionadas con las TIC en los centros educativos; "Desarrollo del personal docente" que requiere información referente a la formación y certificación de docentes para utilizar las TIC con fines educativos. El dominio 
conceptual para la "e-intensidad" es el "Uso de TIC", que promueve el desarrollo de indicadores de la utilización de las TIC en la enseñanza aprendizaje fundamentada en el diseño curricular. Los dominios conceptuales para el desarrollo de indicadores del "eimpacto" son: "Participación, competencias y rendimiento" que sugiere información sobre el número de alumnos graduados en áreas de estudio relacionadas con TIC de naturaleza específica o genérica"; "Resultados e impacto" que indica información sobre el efecto positivo del uso de las TIC en la enseñanza tradicional, desempeño escolar, destrezas laborales, oportunidades de aprendizaje extraescolares y en la administración y gestión escolar"; y "Equidad" que sugiere indicadores sobre los beneficios en igualdad de género y el acceso a la educación en zonas marginadas.

\section{La propuesta metodológica:}

La propuesta para la evaluación de la incorporación de las TIC en la educación superior se fundamenta en dos elementos: 1) el comportamiento organizacional postimplementación -vía la aceptación, rutinización e infusión tecnológicas- de Saga y Zmud (1994) junto con el concepto de la apropiación tecnológica; y, 2) el modelo conceptual para el desarrollo de indicadores TIC de la UNESCO (2009).

La convergencia teórica del comportamiento organizacional post-implementación y de la apropiación tecnológica se explica a continuación.

- En cuanto a su objetivo. El primero estudia la relación de la tecnología con los individuos en el contexto de una organización mientras que la segunda estudia la relación de la tecnología con los individuos en su vida cotidiana.

- En cuanto al enfoque progresivo: el primero considera tres niveles progresivos: la aceptación, la rutinización y la infusión (Saga \& Zmud, 1994) mientras que la segunda identifica igualmente tres dimensiones incrementales: acceso, uso y apropiación (Davis, 1986; Crovi, 2009; Van Dijk, 2005; Colás \& Rodríguez, 2005; Pimienta, 2007; INFOTEC, 2014).

- en cuanto a la aproximación conceptual por cada fase respectiva:

- Respecto de la "aceptación tecnológica" y el "acceso". Los factores que explican a la aceptación ("actitudes", "intenciones" y "frecuencia de uso") expresan el desglose del acto voluntario. Por su lado, el "acceso" se explica a partir de la motivación (Van Dijk, 2005) -uso por el beneficio esperado-, el uso voluntario (INFOTEC, 2014), la conectividad tecnológica (Cobo, 2008; Crovi, 2009; VanDijk, 2005), el acceso a contenidos digitales (Cobo, 2008) y el desarrollo de habilidades digitales e informacionales (Van Dijk, 2005). Se asume que las "actitudes" e "intenciones" -acto evaluativo y posibilidad de uso- comprenden a la "motivación" (de la apropiación); que la "frecuencia de uso" requiere -como lo propone la apropiación tecnológica- la disponibilidad de conectividad; el desarrollo de habilidades para el uso de una computadora y las técnicas para el discernimiento de la información. Aunque el constructo de la aceptación tecnológica implica relaciones más complejas, se asume que la "aceptación" y el "acceso" son convergentes y complementarios;

○ Respecto de la "rutinización" y el "uso". La rutinización se explica a partir de los factores de "desarrollo de infraestructura administrativa", "uso estandarizado" y "uso percibido como normal". Por su parte, el "uso" refiere a la utilización de la tecnología una vez que tiene acceso y se han 
desarrollado habilidades digitales e informacionales. Aunque la "rutinización" se explica mediante mayor complejidad y número de relaciones en el contexto de una organización que el "uso" en la vida cotidiana, la convergencia entre ambos conceptos es evidente;

- Respecto de la "infusión" y la "apropiación". La infusión se explica a partir del "uso emergente"; "uso integrado" y "uso extendido". Por su lado, la "apropiación" se describe como la adaptación de la tecnología a la vida diaria, al trabajo, al aprendizaje y a la generación de conocimiento, a la práctica social del individuo en consonancia con su entorno (INFOTEC, 2014; Pimienta, 2007; Crovi, 2009; Cobo 2008). Al igual que en los casos anteriores, aunque la infusión se explica a partir de relaciones más complejas, se considera que entre la "infusión" y la "apropiación" existe complementariedad y convergencia si se toma en cuenta sus respectivos entornos: el organizacional y el cotidiano.

Respecto de la consistencia de los marcos teóricos del comportamiento organizacional post-implementación (Saga \& Zmud, 1994) y de la apropiación tecnológica (Davis, 1986; Crovi, 2009; Van Dijk, 2005; Colás \& Rodríguez, 2005; Pimienta, 2007; INFOTEC, 2014) con la propuesta de la UNESCO (2009), se analiza tal hecho por cada nivel: por ejemplo, la consistencia entre la "aceptación", el "acceso" y la "e-aptitud", y así sucesivamente.

Tabla 2. Consistencia de la aceptación tecnológica (Saga \& Zmud, 1994) y el "acceso" de la apropiación tecnológica con la e-aptitud (UNESCO, 2009)

\begin{tabular}{|c|c|c|c|}
\hline \multicolumn{2}{|c|}{$\begin{array}{c}\text { Comportamiento organizacional } \\
\text { post-implementación tecnológica } \\
\text { (Saga y Zmud, 1994) }\end{array}$} & \multicolumn{2}{|c|}{$\begin{array}{c}\text { Apropiación tecnológica } \\
\text { (Cobo, 2008; Crovi, 2009; Van Dijk, 2005; } \\
\text { INFOTEC, 2014) }\end{array}$} \\
\hline Nivel & Variables & Nivel & Conceptos \\
\hline $\begin{array}{l}\text { Aceptación } \\
\text { tecnológica }\end{array}$ & $\begin{array}{l}\text { Actitudes } \\
\mathrm{e} \\
\text { Intenciones }\end{array}$ & \multirow{4}{*}{ Acceso } & $\begin{array}{l}\text { Motivación como detonante del interés } \\
\text { (Van Dijk, 2005) } \\
\text { Utilización de manera voluntaria a } \\
\text { partir de una necesidad (INFOTEC, } \\
\text { 2014) }\end{array}$ \\
\hline \multirow[t]{3}{*}{$\begin{array}{l}\text { Acto de recibir el } \\
\text { uso de la } \\
\text { tecnología } \\
\text { voluntariamente }\end{array}$} & \multirow{3}{*}{$\begin{array}{l}\text { Frecuencia } \\
\text { de uso }\end{array}$} & & $\begin{array}{l}\text { Conexión tecnológica (Cobo 2008; } \\
\text { Crovi, 2009; Van Dijk, 2005) } \\
\text { Acceso a contenidos (Cobo, 2008) }\end{array}$ \\
\hline & & & $\begin{array}{c}\text { Entrenamiento en habilidades } \\
\text { informáticas (Cobo, 2008; Van Dijk, } \\
\text { 2005) }\end{array}$ \\
\hline & & & $\begin{array}{c}\text { Desarrollo de habilidades } \\
\text { informacionales (Van Dijk, 2005) }\end{array}$ \\
\hline
\end{tabular}

\begin{tabular}{cc}
\hline \multicolumn{2}{c}{$\begin{array}{c}\text { Marco conceptual para el diseño de } \\
\text { indicadores TIC en la educación } \\
\text { (UNESCO) }\end{array}$} \\
\hline $\begin{array}{c}\text { Nivel de } \\
\text { incorporación }\end{array}$ & $\begin{array}{c}\text { Dominios } \\
\text { conceptuales }\end{array}$ \\
\hline & $\begin{array}{c}\text { Compromiso } \\
\text { político }\end{array}$ \\
\cline { 2 - 2 } e- aptitud & $\begin{array}{c}\text { Asociación } \\
\text { público-privada }\end{array}$ \\
\cline { 2 - 2 } $\begin{array}{c}\text { Disposición de } \\
\text { la entidad } \\
\text { educativa para } \\
\text { beneficiarse de } \\
\text { las TIC }\end{array}$ & Infraestructura \\
& \\
\cline { 2 - 2 } & \\
& $\begin{array}{c}\text { Desarrollo del } \\
\text { personal docente }\end{array}$ \\
& \\
&
\end{tabular}

La Tabla 2 muestra la consistencia entre la "aceptación" y el "acceso" con la "eaptitud". En principio, se asume que la disposición de la entidad educativa para beneficiarse de las TIC (e-aptitud) aterriza el uso voluntario (aceptación) y motivado de la tecnología con la infraestructura de comunicaciones y de capacitación necesarias (acceso). Por consiguiente, se considera que los dominios "Compromiso político" y "Asociación público-privada" - que atienden a la política digital e inversión para favorecer la incorporación de las TIC a la enseñanza aprendizaje- operacionalizan 
inicialmente las "actitudes" e "intenciones" de la "aceptación" y la "motivación" (Van Dijk, 2005) y "uso voluntario" (INFOTEC, 2014) del "acceso". Igualmente, se considera que los dominios conceptuales "Desarrollo del personal docente" e "Infraestructura" operacionalizan inicialmente la "frecuencia de uso" de la "aceptación" y la conexión tecnológica (Cobo, 2008; Crovi, 2009; Van Dijk, 2005), el desarrollo de habilidades digitales (Cobo, 2008) e informacionales (Van Dijk, 2005) y acceso a contenidos (Cobo, 2008) del "acceso". El desarrollo de habilidades digitales e informacionales docentes y la infraestructura de comunicaciones y conectividad son condición indispensable para la "frecuencia de uso" en la enseñanza aprendizaje, donde interesa la disponibilidad de personal docente que adapta sus competencias pedagógicas a un modelo de enseñanza mediado por TIC.

Por su lado, la Tabla 3 presenta la consistencia de los constructos teóricos de la "rutinización" y el "uso" de la apropiación tecnológica con la "e-intensidad". Se asume que en la medida en que se desarrolla la producción de contenidos digitales y la enseñanza mediada por TIC en la entidad educativa (e-intensidad) se institucionaliza el uso de las TIC ("rutinización" y "uso") en la enseñanza aprendizaje. Por consiguiente, se asume que el dominio conceptual "Uso" operacionaliza el "desarrollo de infraestructura administrativa" -como equivalente de la incorporación de la enseñanza en y vía TIC al currículo- y los usos "estandarizado" y "normalizado" (de la rutinización) y el "uso" (apropiación tecnológica) de las TIC en la enseñanza aprendizaje.

Tabla 3. Consistencia de la rutinización tecnológica (Saga \& Zmud, 1994) y la apropiación tecnológica con la e-intensidad (UNESCO, 2009)

\begin{tabular}{cccc}
\hline $\begin{array}{c}\text { Comportamiento organizacional post- } \\
\text { implementación tecnológica } \\
\text { (Saga y Zmud, 1994) }\end{array}$ & $\begin{array}{c}\text { Apropiación tecnológica } \\
\text { (Crovi, 2009; Van Dijk, 2005) }\end{array}$ \\
\hline Nivel & Variables & Nivel & Conceptos \\
\hline $\begin{array}{c}\text { Resarrollo de } \\
\text { Rutinización } \\
\text { tecnológica } \\
\begin{array}{c}\text { Institucionalización } \\
\text { administrativa }\end{array}\end{array}$ & $\begin{array}{c}\text { Uso } \\
\text { de tecnología }\end{array}$ & Ustandarizado & (Crovi, 2009; Van Dijk, 2005) \\
& Uso percibido \\
& "normal" & & \\
\hline
\end{tabular}

\begin{tabular}{|c|c|}
\hline \multicolumn{2}{|c|}{$\begin{array}{l}\text { Marco conceptual para el diseño de } \\
\text { indicadores TIC en la educación } \\
\text { (UNESCO) }\end{array}$} \\
\hline $\begin{array}{c}\text { Nivel de } \\
\text { incorporación }\end{array}$ & $\begin{array}{c}\text { Dominios } \\
\text { conceptuales }\end{array}$ \\
\hline e - intensidad & \\
\hline $\begin{array}{l}\text { Desarrollo de } \\
\text { contenidos } \\
\text { digitales y } \\
\text { gestión de } \\
\text { enseñanza } \\
\text { innovadora y } \\
\text { autoaprendizaje }\end{array}$ & $\begin{array}{c}\text { Uso } \\
\text { (en la enseñanza } \\
\text { aprendizaje } \\
\text { curricular) }\end{array}$ \\
\hline
\end{tabular}

La Tabla 4 muestra la consistencia entre la "infusión" y la "apropiación" con el "eimpacto". Se asume que los efectos académicos, laborales y económicos asociados a las TIC (e-impacto) indican la incrustación de la tecnología en los procesos de enseñanza aprendizaje (infusión) y el impacto en la cotidianidad (apropiación). En consecuencia, se considera que los dominios "Participación, competencias y rendimiento"-graduación en áreas relacionadas con TIC-; "Resultados e impacto" -efecto en la enseñanza, desempeño académico y laboral y gestión escolar-; y "Equidad" -igualdad en género y acceso- operacionalizan inicialmente los usos "emergente", "integrado" y "extendido" de la "rutinización" y la integración de la tecnología a la práctica social de la "apropiación". Se entiende que la graduación de especialistas TIC -como manifestación de la incorporación formal de las TIC en el modelo educativo- evidencia el "uso integrado" de las TIC. El efecto en la enseñanza aprendizaje, el logro académico y laboral, entre otros, implica el uso "integrado" y "extendido" de las TIC y base del 
aprendizaje. El favorecimiento de la enseñanza aprendizaje en género y acceso democrático con énfasis en zonas marginadas alude a los usos "integrado" y "extendido" de la tecnología y el impacto social.

Tabla 4. Consistencia de la infusión (Saga \& Zmud, 1994) y la apropiación tecnológica con el e-impacto (UNESCO, 2009)

\begin{tabular}{|c|c|c|c|c|c|}
\hline \multicolumn{2}{|c|}{$\begin{array}{c}\text { Comportamiento } \\
\text { organizacional post- } \\
\text { implementación tecnológica } \\
(\text { Saga \& Zmud, 1994) } \\
\end{array}$} & \multicolumn{2}{|c|}{$\begin{array}{c}\text { Apropiación tecnológica } \\
\text { (Cobo, 2008; Crovi, 2009; Overdijik \& Van Diggelen, } \\
\text { 2006; Pimienta, 2007; Colás \& Rodríguez, 2005; } \\
\text { INFOTEC, 2014) }\end{array}$} & \multicolumn{2}{|c|}{$\begin{array}{l}\text { Marco conceptual para el } \\
\text { diseño de indicadores TIC en } \\
\text { la educación } \\
\text { (UNESCO) }\end{array}$} \\
\hline Nivel & Variables & Nivel & Conceptos & $\begin{array}{l}\text { Nivel de } \\
\text { incorporació } \\
n\end{array}$ & $\begin{array}{l}\text { Dominios } \\
\text { conceptuales }\end{array}$ \\
\hline \multirow{5}{*}{$\begin{array}{c}\text { Infusión } \\
\text { tecnológica } \\
\text { Incrustación } \\
\text { profunda de } \\
\text { la tecnología } \\
\text { y sistemas de } \\
\text { trabajo de la } \\
\text { organización }\end{array}$} & & \multirow{5}{*}{ Apropiación } & $\begin{array}{l}\text { Adopción y adaptación tecnológica a la } \\
\text { vida diaria, prácticas y rutinas de trabajo } \\
\text { de un individuo (INFOTEC, 2014) }\end{array}$ & \multirow{5}{*}{$\begin{array}{c}\text { e-impacto } \\
\text { Efectos en } \\
\text { el } \\
\text { desempeño } \\
\text { académico y } \\
\text { laboral, } \\
\text { productivida } \\
\text { d económica } \\
\text { y } \\
\text { aprendizaje } \\
\text { permanente }\end{array}$} & $\begin{array}{l}\text { Participación, } \\
\text { competencias } \\
\text { y rendimiento }\end{array}$ \\
\hline & $\begin{array}{c}\text { Uso } \\
\text { emergente }\end{array}$ & & $\begin{array}{l}\text { Integración de las TIC a la práctica social } \\
\text { (Crovi, 2009) }\end{array}$ & & $\begin{array}{l}\text { Resultados e } \\
\text { impacto }\end{array}$ \\
\hline & $\begin{array}{c}\text { Uso } \\
\text { integrado }\end{array}$ & & $\begin{array}{l}\text { Proceso de construcción social en el que } \\
\text { las acciones y pensamientos son } \\
\text { impactados por la tecnología (Overdijik } \\
\text { \& Van Diggelen, 2006) }\end{array}$ & & \multirow{3}{*}{ Equidad } \\
\hline & $\begin{array}{l}\text { Uso } \\
\text { extendido }\end{array}$ & & $\begin{array}{c}\text { Forma en que son asumidas las TIC para } \\
\text { transformar la interpretación de la } \\
\text { realidad y base del aprendizaje (Colás \& } \\
\text { Rodríguez, 2005) }\end{array}$ & & \\
\hline & & & $\begin{array}{l}\text { Colaboración entre usuarios para el } \\
\text { aprendizaje y generación de nuevo } \\
\text { conocimiento (Cobo, 2008) }\end{array}$ & & \\
\hline
\end{tabular}

El análisis de la consistencia teórico-metodológica anterior permite asumir que: a) los dominios conceptuales de la propuesta de la UNESCO (2009) operacionalizan de manera inicial el marco teórico del comportamiento organizacional postimplementación tecnológica de Saga y Zmud (1994) y la apropiación tecnológica cuando se orienta hacia una organización de carácter educativo; y que, b) por consiguiente, los indicadores definidos para cada dominio conceptual de la UNESCO (2009) constituyen un sistema de indicadores inicial que operacionalizan los constructos teóricos de la aceptación, rutinización, infusión y apropiación tecnológicas en la educación.

De acuerdo a Mintzberg, citado en Duarte y Ventura (2011) las instituciones de educación superior son organizaciones complejas -en coincidencia con Clark (1983)con múltiples centros de poder de decisión que conlleva un rango amplio de intereses heterogéneos. La universidad es una organización diversa e interactuante con su entorno (Dettmer, 2004; Clark, 1986; Neave, 1986; Vught, 1986); y división del trabajo fundamentada en la diferenciación del conocimiento profesional. Entonces, se considera pertinente el análisis de la incorporación tecnológica universitaria desde el comportamiento organizacional de Saga y Zmud (1994) y de la apropiación tecnológica (Davis, 1986; Crovi, 2009; Van Dijk, 2005; Colás \& Rodríguez, 2005; Pimienta, 2007; INFOTEC, 2014). 


\section{Primera aproximación al sistema de indicadores:}

Los indicadores TIC de la metodología UNESCO conforman una base valiosa para la propuesta metodológica. Para el diseño de la batería de indicadores se sigue el procedimiento siguiente:

1. Adaptación de los dominios conceptuales de indicadores al contexto universitario

2. Selección de indicadores de los dominios conceptuales de la UNESCO (2009) bajo dos criterios: 1) los correspondientes a educación superior: niveles CINE (Clasificación Internacional Normalizada de la Educación) 5, 6, 7, y 8 (UNESCO, 2009; 2011); 2) los que tiene pertinencia desde la perspectiva teórica tratada.

3. Adaptación de los indicadores seleccionados.

4. Revisión de estudios internacionales coincidentes con la metodología de la UNESCO (2009): Web Index 2012, 2013 y 2014 de la WWWF (2012; 2013; 2014), el Networked Readiness Index 2015 del WEF (2015); el Índice de Desarrollo de las TIC 2014 de la UIT (2014); y los Indicadores Clave sobre TIC de los Partnerships para la Medición de las TIC para el Desarrollo (PMTD) (UIT, 2010).

5. Revisión de trabajos de investigación relevantes basados en el marco teórico del comportamiento organizacional post-implementación y apropiación tecnológicas.

6. Si es el caso, diseñar indicadores adicionales por demanda del marco teórico.

De esta forma, se propone un marco metodológico para analizar la incorporación de las TIC en la educación superior desde el comportamiento organizacional postimplementación tecnológica con tres fases progresivas: aceptación, rutinización e infusión. La aceptación se analiza desde 4 dominios conceptuales y 33 indicadores; la rutinización considera 1 dominio conceptual con 8 indicadores; la infusión contempla 3 dominios y 17 indicadores. El sistema total se compone de 58 indicadores (Tabla 5).

Tabla 5. Sistema de indicadores para medir la incorporación tecnológica

\begin{tabular}{|c|c|c|c|}
\hline \multicolumn{4}{|c|}{ Análisis del comportamiento organizacional pos implementación tecnológica universitaria } \\
\hline Dimensión & Dominio conceptual & No. de indicadores & Totales \\
\hline \multirow{4}{*}{ Aceptación tecnológica } & Compromiso político & 7 & \multirow{4}{*}{33} \\
\hline & Asociación público-privada & 5 & \\
\hline & Infraestructura & 11 & \\
\hline & Desarrollo de personal docente & 10 & \\
\hline Rutinización tecnológica & Uso & 8 & 8 \\
\hline \multirow{4}{*}{ Infusión tecnológica } & Participación, competencias y rendimiento & $\overline{6} 6$ & \multirow{3}{*}{17} \\
\hline & Resultados e impacto & 7 & \\
\hline & Equidad & 4 & \\
\hline & Total & & 58 \\
\hline
\end{tabular}

\section{1.- Indicadores para la aceptación tecnológica}

Compromiso político

Políticas, programas o marcos normativos universitarios que sustenten y orienten la implementación de la TIC en la enseñanza aprendizaje (Tabla 6).

Tabla 6. Indicadores para medir el compromiso político de la aceptación tecnológica universitaria 


\begin{tabular}{|c|c|c|c|c|c|c|}
\hline \multicolumn{2}{|c|}{ Fundamento } & \multirow[t]{2}{*}{$\#$} & \multirow{2}{*}{$\begin{array}{c}\text { Fuente } \\
\text { WWWF (2012) }\end{array}$} & \multirow{2}{*}{$\begin{array}{c}\begin{array}{c}\text { Etiqueta } \\
\text { fuente }\end{array} \\
\text { WEFG }\end{array}$} & \multirow{2}{*}{$\begin{array}{l}\text { Etiqueta } \\
\text { ATCP01 }\end{array}$} & \multirow{2}{*}{$\begin{array}{l}\text { Indicador } \\
\text { Políticas sobre la importancia de las TIC para la visión de } \\
\text { futuro de la universidad }\end{array}$} \\
\hline \multirow{7}{*}{ 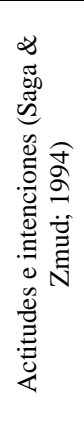 } & \multirow{7}{*}{ 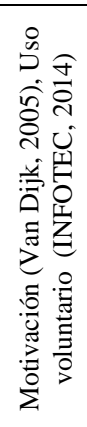 } & & & & & \\
\hline & & 2 & WWWF (2012) & Q16 & ATCP02 & $\begin{array}{l}\text { Estudios sobre la calidad de la formación de especialistas } \\
\text { TIC }\end{array}$ \\
\hline & & 3 & UNESCO (2009) & ED12 & ATCP03 & $\begin{array}{l}\text { Porcentaje del gasto total en TIC en educación } \\
\text { correspondiente al gasto corriente en TIC en educación }\end{array}$ \\
\hline & & 4 & UNESCO (2009) & ED13 & ATCP04 & $\begin{array}{l}\text { Porcentaje del gasto total en TIC en educación } \\
\text { correspondiente al gasto de capital en TIC en educación }\end{array}$ \\
\hline & & 5 & UNESCO (2009) & ED14 & ATCP05 & $\begin{array}{l}\text { Porcentaje del gasto total correspondiente al gasto } \\
\text { corriente en TIC en educación }\end{array}$ \\
\hline & & 6 & UNESCO (2009) & ED15 & ATCP06 & $\begin{array}{l}\text { Porcentaje del gasto total correspondiente al gasto de } \\
\text { capital en TIC en educación }\end{array}$ \\
\hline & & 7 & UNESCO (2009) & ED16 & ATCP07 & Gasto promedio en TIC en educación por alumno \\
\hline
\end{tabular}

\section{Asociación público-privada}

Inversión pública en alianza con fondos privados para la implementación de las TIC en el proceso de enseñanza aprendizaje (Tabla 7).

Tabla 7. Indicadores para medir la asociación público-privada de la aceptación tecnológica universitaria

\begin{tabular}{|c|c|c|c|c|c|c|}
\hline \multicolumn{2}{|c|}{ Fundamento } & $\#$ & Fuente & $\begin{array}{l}\text { Etiqueta } \\
\text { fuente }\end{array}$ & Etiqueta & Indicador \\
\hline \multirow{5}{*}{ 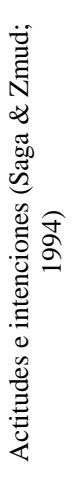 } & \multirow{5}{*}{ 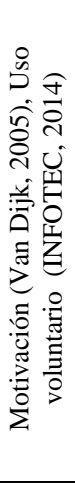 } & 1 & UNESCO (2009) & ED17 & ATAP01 & $\begin{array}{l}\text { Porcentaje del gasto corriente total en TIC en educación } \\
\text { correspondiente al gasto corriente privado en TIC en } \\
\text { educación }\end{array}$ \\
\hline & & 2 & UNESCO (2009) & ED18 & ATAP02 & $\begin{array}{l}\text { Porcentaje del gasto de capital total en TIC en educación } \\
\text { correspondiente al gasto privado de capital en TIC en } \\
\text { educación }\end{array}$ \\
\hline & & 3 & UNESCO (2009) & ED19 & ATAP03 & $\begin{array}{l}\text { Porcentaje de gasto corriente total en TIC en educación } \\
\text { correspondiente al gasto corriente extranjero en TIC en } \\
\text { educación }\end{array}$ \\
\hline & & 4 & UNESCO (2009) & ED20 & ATAP04 & $\begin{array}{l}\text { Porcentaje de gasto de capital total en TIC en educación } \\
\text { correspondiente al gasto extranjero de capital en TIC en } \\
\text { educación }\end{array}$ \\
\hline & & 5 & UNESCO (2009) & ED21 & ATAP05 & $\begin{array}{l}\text { Porcentaje del gasto corriente total en TIC en educación } \\
\text { correspondiente al gasto total corriente privado y } \\
\text { extranjero en TIC en educación }\end{array}$ \\
\hline
\end{tabular}

\section{Infraestructura}

Cantidad y calidad de instalaciones o recursos relacionados TIC disponibles en escuelas o facultades para la enseñanza aprendizaje (Tabla 8).

Tabla 8. Indicadores para medir la infraestructura de la aceptación tecnológica universitaria

\begin{tabular}{|c|c|c|c|c|c|c|}
\hline Funda & amento & $\#$ & Fuente & $\begin{array}{l}\text { Etiqueta } \\
\text { fuente }\end{array}$ & Etiqueta & Indicador \\
\hline \multirow{6}{*}{ 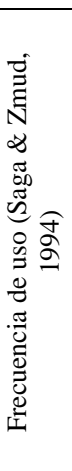 } & \multirow{6}{*}{ 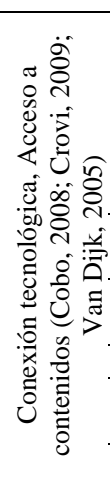 } & 1 & UNESCO (2009) & ED5* & ATIN01 & $\begin{array}{l}\text { Porcentaje de escuelas o facultades que cuentan con } \\
\text { acceso a Internet por tipo: } \\
\text { - Cualquier tipo; } \\
\text { - Banda estrecha fija (mediante cable modem, ISDN); } \\
\text { - Banda ancha fija (DSL, cable, otras); } \\
\text { - Banda ancha y estrecha fijas }\end{array}$ \\
\hline & & 2 & UNESCO (2009) & ED24 & ATIN02 & $\begin{array}{l}\text { Porcentaje de escuelas o facultades con licencias o } \\
\text { subscripciones a bibliotecas científicas digitales }\end{array}$ \\
\hline & & 3 & UNESCO (2009) & ED24bis & ATIN03 & $\begin{array}{l}\text { Porcentaje de escuelas o facultades con licencias o } \\
\text { subscripciones a laboratorios experimentales virtuales }\end{array}$ \\
\hline & & 4 & UNESCO (2009) & ED25* & ATIN04 & Relación alumnos/computadoras con conexión a Internet \\
\hline & & 5 & UNESCO (2009) & ED27 & ATIN05 & $\begin{array}{l}\text { Número promedio de computadoras con conexión a } \\
\text { Internet por escuela o facultad }\end{array}$ \\
\hline & & 6 & UNESCO (2009) & ED28 & ATIN06 & Porcentaje de computadoras de propiedad de los alumnos \\
\hline
\end{tabular}




\begin{tabular}{ccccl}
\hline 7 & UNESCO (2009) & ED29 & ATIN07 & $\begin{array}{l}\text { disponibles para uso pedagógico } \\
\text { pedagógico de todas las computadoras disponibles para uso }\end{array}$ \\
\hline 8 & UNESCO (2009) & ED30 & ATIN08 & $\begin{array}{l}\text { Porcentaje de todas las computadoras disponibles para uso } \\
\text { administrativo }\end{array}$ \\
\hline 9 & UNESCO (2009) & ED32 & ATIN09 & $\begin{array}{l}\text { Porcentaje de escuelas o facultades que cuentan con un } \\
\text { sitio Web que permite hospedar (host) páginas blog de } \\
\text { propiedad de alumnos y docentes }\end{array}$ \\
\hline 10 & UNESCO (2009) & ED33 & ATIN10 & $\begin{array}{l}\text { Porcentaje de escuelas o facultades que ofrecen } \\
\text { programas de educación a distancia mediados por TIC }\end{array}$ \\
\hline 11 & WWWF (2012) & Q18 & ATIN11 & $\begin{array}{l}\text { Proporción de escuelas o facultades que cuentan con } \\
\text { suministro de energía eléctrica }\end{array}$ \\
\hline
\end{tabular}

"Indicador definido originalmente para niveles CINE 1-3: primaria, secundaria baja y secundaria alta

\section{Desarrollo del personal docente}

Formación y asignación de docentes certificados en habilidades digitales e informacionales para utilizar las TIC en la enseñanza aprendizaje (Tabla 9).

Tabla 9. Indicadores para medir el desarrollo del personal docente de la aceptación tecnológica universitaria

\begin{tabular}{|c|c|c|c|c|c|c|}
\hline \multicolumn{2}{|c|}{ Fundamento } & $\#$ & Fuente & $\begin{array}{c}\text { Etiqueta } \\
\text { fuente }\end{array}$ & Etiqueta & Indicador \\
\hline \multirow{10}{*}{ 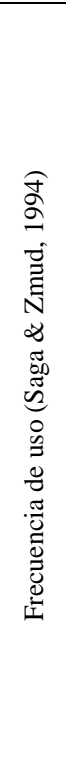 } & \multirow{10}{*}{ 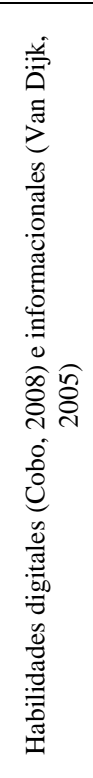 } & 1 & UNESCO (2009) & ED8* & ATDD01 & $\begin{array}{l}\text { Porcentaje de docentes certificados para enseñar } \\
\text { conocimientos básicos computacionales }\end{array}$ \\
\hline & & 2 & $\begin{array}{c}\text { (UNESCO, 2009; } \\
\text { CAUL, 2001; ACRL, } \\
2000 ; \\
\text { ANZIIL, 2004) } \\
\end{array}$ & ED8b* & ATDD02 & $\begin{array}{l}\text { Porcentaje de docentes certificados para enseñar } \\
\text { conocimientos básicos informacionales }\end{array}$ \\
\hline & & 3 & UNESCO (2009) & ED34* & ATDD03 & $\begin{array}{l}\text { Porcentaje de escuelas que cuentan con servicios de } \\
\text { soporte técnico TIC }\end{array}$ \\
\hline & & 4 & UNESCO (2009) & ED35* & ATDD04 & $\begin{array}{l}\text { Porcentaje de docentes certificados mediante programas } \\
\text { de educación a distancia mediados por TIC }\end{array}$ \\
\hline & & 5 & UNESCO (2009) & ED36* & ATDD05 & $\begin{array}{l}\text { Porcentaje de docentes que enseñan conocimientos } \\
\text { básicos computacionales }\end{array}$ \\
\hline & & 6 & $\begin{array}{c}\text { (UNESCO, 2009; } \\
\text { CAUL, 2001; ACRL, } \\
2000 ; \\
\text { ANZIIL, 2004) }\end{array}$ & ED36bis & ATDD06 & $\begin{array}{l}\text { Porcentaje de docentes que enseñan conocimientos } \\
\text { básicos informacionales }\end{array}$ \\
\hline & & 7 & UNESO (2009) & ED37* & ATDD07 & $\begin{array}{l}\text { Porcentaje de docentes que enseñan una o varias } \\
\text { asignaturas usando recursos TIC }\end{array}$ \\
\hline & & 8 & UNESCO (2009) & ED38* & ATDD08 & $\begin{array}{l}\text { Porcentaje de docentes certificados para enseñar una o } \\
\text { varias asignaturas escolares utilizando recursos TIC }\end{array}$ \\
\hline & & 9 & UNESCO (2009) & ED39* & ATDD09 & $\begin{array}{l}\text { Relación alumnos/docentes del área de conocimientos } \\
\text { básicos computacionales (o informática) }\end{array}$ \\
\hline & & 10 & UNESCO (2009) & ED40* & ATDD10 & $\begin{array}{l}\text { Relación alumnos/docentes que utilizan TIC para la } \\
\text { enseñanza }\end{array}$ \\
\hline
\end{tabular}

\section{2.- Indicadores para la rutinización tecnológica}

\section{Uso de TIC en la enseñanza aprendizaje}

Grado de integración de las TIC en el currículo y acceso a las TIC en las escuelas o facultades (Tabla 10).

Tabla 10. Indicadores para medir el uso de la rutinización tecnológica universitaria

\begin{tabular}{llcccl}
\hline Fundamento & $\#$ & Fuente & $\begin{array}{c}\text { Etiqueta } \\
\text { fuente }\end{array}$ & Etiqueta & Indicador \\
\hline & UNESCO (2009) & ED6* & RTEA01 & $\begin{array}{l}\text { Porcentaje de alumnos que cuentan con acceso a internet } \\
\text { en las distintas facultades y escuelas }\end{array}$ \\
\hline & UNESCO (2009) & ED41* & RTEA02 & $\begin{array}{l}\text { Porcentaje de alumnos con derecho a usar los laboratorios } \\
\text { computacionales de las facultades o escuelas como medio } \\
\text { auxiliar de enseñanza }\end{array}$ \\
\hline
\end{tabular}




\begin{tabular}{|c|c|c|c|c|}
\hline 3 & UNESCO (2009) & ED42 & RTEA03 & $\begin{array}{l}\text { Porcentaje de escuelas y facultades que proporcionan } \\
\text { correo electrónico a todo el personal docente }\end{array}$ \\
\hline 4 & UNESCO (2009) & ED43 & RTEA04 & $\begin{array}{l}\text { Porcentaje de escuelas y facultades que proporcionan una } \\
\text { cuenta de correo electrónico a todos sus alumnos }\end{array}$ \\
\hline 5 & $\begin{array}{l}\text { República de Corea y } \\
\text { Egipto, citado en } \\
\text { (UNESCO, 2009) }\end{array}$ & EDxx & RTEA05 & $\begin{array}{l}\text { Porcentaje de escuelas y facultades especializadas en } \\
\text { áreas de TIC o capacitación en TIC }\end{array}$ \\
\hline 6 & $\begin{array}{l}\text { UNESCO, Bangkok, } \\
\text { citado en UNESCO } \\
\text { (2009) }\end{array}$ & EDxx & RTEA06 & $\begin{array}{l}\text { Porcentaje de escuelas y facultades que cuentan con } \\
\text { software educativo producido por la universidad para la } \\
\text { enseñanza de asignaturas escolares }\end{array}$ \\
\hline 7 & $\begin{array}{l}\text { UNESCO, Bangkok, } \\
\text { citado en UNESCO } \\
(2009)\end{array}$ & EDxx & RTEA07 & $\begin{array}{l}\text { Porcentaje de escuelas y facultades que cuentan con } \\
\text { software educativo para la enseñanza de habilidades } \\
\text { básicas de computación producido fuera de la universidad }\end{array}$ \\
\hline 8 & $\begin{array}{l}\text { Belarús, citado en } \\
\text { UNESCO (2009) }\end{array}$ & EDxx & RTEA08 & $\begin{array}{l}\text { Porcentaje de escuelas y facultades que cuentan por lo } \\
\text { menos con una unidad de software especializado: } \\
\text { - uso administrativo } \\
\text { - biblioteca escolar } \\
\text { - enseñanza en ciencias básicas } \\
\text { - enseñanza de ciencias naturales } \\
\text { - enseñanza de asignaturas humanísticas }\end{array}$ \\
\hline
\end{tabular}

*Indicador definido originalmente para niveles CINE 1-3: primaria, secundaria baja y secundaria alta

\section{3.- Indicadores para la infusión tecnológica}

\section{Participación, competencias y rendimiento}

Número de alumnos graduados en áreas de estudio de naturaleza específica o genérica relacionadas a las TIC (Tabla 11).

Tabla 11. Indicadores para medir la participación, competencias y rendimiento de la infusión tecnológica universitaria

\begin{tabular}{|c|c|c|c|c|c|c|}
\hline \multicolumn{2}{|c|}{ Fundamento } & \# & Fuente & $\begin{array}{c}\text { Etiqueta } \\
\text { fuente }\end{array}$ & Etiqueta & Indicador \\
\hline \multirow{6}{*}{ 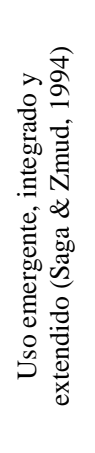 } & \multirow{6}{*}{ 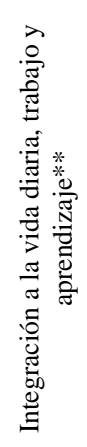 } & 1 & UNESCO (2009) & ED7 & ITPC01 & $\begin{array}{l}\text { Porcentaje de alumnos (por género) matriculados en } \\
\text { carreras relacionadas con TIC }\end{array}$ \\
\hline & & 2 & UNESCO (2009) & ED44* & ITPC02 & $\begin{array}{l}\text { Porcentaje de alumnos matriculados en grados que } \\
\text { ofrecen enseñanza asistida por TIC }\end{array}$ \\
\hline & & 3 & UNESCO (2009) & ED45* & ITPC03 & $\begin{array}{l}\text { Porcentaje de alumnos matriculados en grados en los que } \\
\text { actualmente se enseñan conocimientos básicos } \\
\text { computacionales }\end{array}$ \\
\hline & & 4 & UNESCO (2009) & ED46 & ITPC04 & $\begin{array}{l}\text { Porcentaje de alumnos (por género) graduados de carreras } \\
\text { relacionadas con las TIC en el último año académico }\end{array}$ \\
\hline & & 5 & UNESCO (2009) & ED47 & ITPC05 & $\begin{array}{l}\text { Porcentaje de alumnos (por género) matriculados en } \\
\text { programas de educación a distancia mediados por TIC }\end{array}$ \\
\hline & & 6 & UNESCO (2009) & ED48* & ITPC06 & $\begin{array}{l}\text { Porcentaje de alumnos que completaron exitosamente un } \\
\text { curso de conocimientos básicos computacionales (o } \\
\text { informática) durante el último año académico }\end{array}$ \\
\hline
\end{tabular}

*Indicador definido originalmente para niveles CINE 1-3: primaria, secundaria baja y secundaria alta

**(INFOTEC, 2014; Pimienta, 2007; Crovi, 2009; Over Dijk \& Van Diggelen, 2006; Colás \& Rodríguez, 2005; Cobo, 2008)

\section{Resultados e impacto}

Efecto positivo de las TIC en la mejora de la enseñanza aprendizaje, gestión y desempeño escolar; formación en destrezas laborales TIC; programas de capacitación para el empleo mediados por TIC fuera del sistema formal de educación (Tabla 12).

Tabla 12. Indicadores para medir los resultados e impacto de la infusión tecnológica universitaria

\begin{tabular}{|c|c|c|c|c|c|}
\hline $\begin{array}{c}\text { Fundament } \\
\text { o }\end{array}$ & $\#$ & Fuente & $\begin{array}{l}\text { Etiqueta } \\
\text { fuente }\end{array}$ & Etiqueta & Indicador \\
\hline 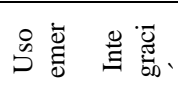 & 1 & UNESCO (2009) & ED49* & ITRI01 & $\begin{array}{l}\text { Tasa de promoción de alumnos que cursan grados que } \\
\text { imparten enseñanza asistida por TIC (por género, } \\
\text { escuela y grado) }\end{array}$ \\
\hline
\end{tabular}

La evaluación de la integración de las TIC en la educación superior: fundamento para una metodología. 


\begin{tabular}{|c|c|c|c|c|}
\hline 2 & UNESCO (2009) & ED50* & ITRI02 & $\begin{array}{l}\text { Tasa de promoción de alumnos que cursan grados que } \\
\text { no imparten enseñanza asistida por TIC (por género, } \\
\text { escuela y grado) }\end{array}$ \\
\hline 3 & UNESCO (2009) & ED51 & ITRI03 & $\begin{array}{l}\text { Tasa de desempeño escolar (por género, escuela y } \\
\text { grado) en la enseñanza asistida por TIC (ED49/ED50) }\end{array}$ \\
\hline 4 & UNESCO (2009) & & ITRI04 & $\begin{array}{l}\text { Tasa anual de cambio del porcentaje de alumnos que } \\
\text { completaron exitosamente un curso de conocimientos } \\
\text { básicos computacionales respecto del año académico } \\
\text { anterior. }\end{array}$ \\
\hline 5 & UNESCO (2009) & & ITRI05 & $\begin{array}{l}\text { Tasa anual de cambio del porcentaje de alumnos (por } \\
\text { género) graduados de carreras relacionadas con las TIC } \\
\text { respecto del último año académico }\end{array}$ \\
\hline 6 & UNESCO (2009) & & ITRI06 & $\begin{array}{l}\text { Tasa anual de cambio del porcentaje de computadoras } \\
\text { disponibles para uso administrativo }\end{array}$ \\
\hline 7 & UNESCO (2009) & & ITRI07 & $\begin{array}{l}\text { Porcentaje de estudiantes matriculados en programas } \\
\text { de extensión o capacitación para el empleo mediados } \\
\text { por TIC fuera del sistema formal de educación }\end{array}$ \\
\hline
\end{tabular}

\section{Equidad}

Impacto de las TIC en equidad de género y en el acceso a la educación en zonas pobres y marginadas (Tabla 13).

Tabla 13. Indicadores para medir la equidad de la infusión tecnológica universitaria

\begin{tabular}{|c|c|c|c|c|c|}
\hline Fundamento & $\#$ & Fuente & $\begin{array}{c}\text { Etiqueta } \\
\text { fuente }\end{array}$ & Etiqueta & Indicador \\
\hline \multirow{4}{*}{ 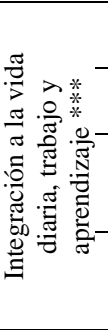 } & 1 & UNESCO (2009) & ED53 & ITEQ01 & $\begin{array}{l}\text { Número de alumnas graduadas en áreas relacionadas con } \\
\text { las TIC por cada } 1000 \text { graduados varones }\end{array}$ \\
\hline & 2 & UNESCO (2009) & ED52 & ITEQ02 & $\begin{array}{l}\text { Porcentaje de escuelas -o facultades- rurales que cuentan } \\
\text { con enseñanza asistida por TIC }\end{array}$ \\
\hline & 3 & $\begin{array}{c}\text { República de Corea, } \\
\text { citado en UNESCO } \\
(2009)\end{array}$ & EDxx & ITEQ03 & $\begin{array}{l}\text { Porcentaje de escuelas que usan software especializado } \\
\text { para alumnos con capacidades diferentes }\end{array}$ \\
\hline & 4 & $\begin{array}{c}\text { República de Corea, } \\
\text { citado en UNESCO } \\
(2009)\end{array}$ & EDxx* & ITEQ04 & $\begin{array}{l}\text { Porcentaje de docentes mujeres que actualmente enseñan } \\
\text { una o varias asignaturas utilizando recursos TIC }\end{array}$ \\
\hline
\end{tabular}

\section{Viabilidad de la propuesta:}

La propuesta metodológica aquí presentada se aplicó como estudio de caso en la Universidad Veracruzana de México (UV). La experiencia obtenida se describe mediante tres aspectos: 1) la recopilación de información; 2) el diseño de indicadores; 3) los resultados obtenidos. Respecto del primero, se recopiló la información de 40 de 58 indicadores -aproximadamente el 70\%-. La información faltante se caracterizó por su especificidad respecto del enfoque tecnológico: por ejemplo, porcentaje de docentes certificados mediante programas de educación a distancia mediados por TIC (indicador ATDD04). La información fue recolectada desde el portal web de la UV (www.uv.mx) lo que facilitó el estudio y se asume que este hecho denota el esfuerzo de la universidad por transparentar ante la sociedad su actividad institucional. La disponibilidad de la información para la evaluación decreció conforme se avanzó a los niveles superiores: 75\% (Aceptación), $62.5 \%$ (Rutinización) y 58.8\% (Infusión), lo que tiene sentido dado que conforme se progresa en niveles superiores de incorporación tecnológica, se tiende a la dificultad por incorporar las TIC de manera profunda en la enseñanza aprendizaje universitaria y en consecuencia de las evidencias. Respecto del segundo aspecto, la viabilidad de los indicadores se considera evidente por encontrar la información 
requerida. En algunos casos, fue necesario manejar información aproximada por la especificidad de los datos demandados por los indicadores: por ejemplo, gasto corriente total en TIC en educación como porcentaje del gasto corriente privado en TIC en educación (indicador ATAP01). Respecto del tercer aspecto, se obtuvo una calificación general de $82.55 \%$, lo que indica un nivel alto de integración de las TIC en la dimensión de la enseñanza aprendizaje de la UV. Esta calificación resulta de las mediciones obtenidas por nivel: 31.85 de 33.33 puntos posibles en "aceptación tecnológica" -con una calificación particular del $95.5 \%$ - que se sustenta en el desarrollo de competencias digitales docentes para fines pedagógicos y el respaldo financiero en alianza públicoprivada para el fortalecimiento de la infraestructura tecnológica, lo que se asume como evidencia del acto institucional voluntario y motivado de usar las TIC para beneficiar la enseñanza aprendizaje y crear las condiciones para ello; 26.64 de 33.33 en "rutinización tecnológica" -con una calificación particular de $80 \%$ - que se sustenta principalmente en el acceso a internet para fines pedagógicos -como correo electrónico, blogs, laboratorios virtuales- de la totalidad de la matrícula -aproximadamente de 79,000 en educación formal y no formal (www.uv.mx/informacionestadistica/files/2014/01/UVNumAgs.pdf)-, lo que se asume como evidencia del grado de incorporación a la vida cotidiana universitaria. En este nivel, el número reducido de indicadores (8) favoreció la calificación alta; y 24.06 de igualmente 33.33 -con una calificación particular de $72.2 \%$ - en "infusión tecnológica" que se sustenta en la cobertura en zonas rurales -UV intercultural en 4 regiones indígenas y de difícil acceso; la visión de equidad de género tanto función docente como en la formación de alumnos; la totalidad de las asignaturas apoyadas por el uso de las TIC; y el reconocimiento del desarrollo de competencias digitales como competencia transversal en el modelo educativo, lo que se asume como evidencia de la penetración de las TIC como componente clave para la formación universitaria. La ausencia de evidencia sobre la formación mediada por TIC se percibe en los documentos institucionales (UV, 2013) como una política institucional que espera a la consolidación de la modalidad presencial.

El cálculo de las calificaciones se realizó con los indicadores con información: el hecho de no contar con los datos de algunos indicadores implicaba efecto negativo en las calificaciones por ausencia de datos lo que no implicaba que no existieran las acciones. Se asume que conforme se cuente con la mayor parte de los datos, mayor consistencia del estudio. La aplicación de la propuesta requirió de la ponderación de los niveles de "aceptación", "rutinización" e "infusión" y de sus indicadores. De acuerdo a los estudios similares, la ponderación de fases atiende dos posturas: 1) la que asigna valor mayor al impacto que a la infraestructura. Por ejemplo, el Web Index 2012 (WWWF, 2012) pondera la disposición (infraestructura) con 20\%; la web (desarrollo y uso de contenidos) con 20\%; y el impacto (político, económico y social) con 60\%, lo que implica que los países con mayor inversión económica no consiguen necesariamente el mejor efecto; 2) en sentido contrario, la que asigna valor mayor a la infraestructura que al impacto. Por ejemplo, el Índice de Desarrollo de las TIC 2014 (UIT, 2014) pondera el "Acceso a las TIC" (infraestructura) con 40\%; la "Utilización de las TIC" (acceso) con $40 \%$; y las "Capacidades de las TIC" (impacto educativo) con 20\%. Se considera que se valora el esfuerzo de los gobiernos por disponer de políticas digitales necesarias y la inversión en infraestructura de comunicaciones por sobre el impacto. Para fines de esta investigación se adoptó la ponderación igualitaria (33.33\%) para niveles e indicadores en razón de la generación de conocimiento inicial de la metodología. Bajo el ejercicio

La evaluación de la integración de las TIC en la educación superior: fundamento para una metodología. Pedro Nolasco Vázquez y Mario Miguel Ojeda Ramírez. 
preliminar realizado, se asume que aunque la ponderación homogénea proporcionó resultados consistentes, es probable que la ponderación heterogénea acorde al contexto y convicción de la universidad pueda rendir mejores resultados.

Por último, la estrategia de corroboración consistió en el análisis comparativo del Programa de Trabajo Estratégico 2013 - 2017 del rectorado actual (UV, 2013) con la batería de indicadores, donde se observa que la demanda de información de los indicadores es consistente con lo propuesto como objetivos y acciones planteadas. Un hallazgo a resaltar es la convicción por disponer de una sección del portal universitario a la divulgación de información sobre planes y actividades de la gerencia universitaria (www.uv.mx/documentos) así como información estadística institucional (www.uv.mx/informacion-estadística) donde se incluye el tema de las TIC.

\section{Conclusiones:}

La medición de la política digital universitaria tiene alta relevancia social al manifestarse las TIC como un instrumento que detona desempeño académico, formación docente, cobertura y calidad educativa en la educación superior (DEGV, 2011; Kozma, 2005; UNESCO, 2009) y ésta a su vez como uno de los pilares del desarrollo humano (UNESCO, 1998; OEI, 1999; UEALC, 2000; Peng \& Wang, 2008).

El progreso del ejercicio evaluativo de la política digital universitaria desde el CMM resulta un logro importante: a mayor madurez digital de los procesos clave de la organización, mayor madurez organizacional (Curtis et al., 1995; Paulk et al., 1993). Sin embargo, aunque esto podría interpretarse como efectividad de la implementación tecnológica no es posible saber si uno de los impedimentos para el avance pudiera originarse en la relación de los individuos con la tecnología en el contexto de la organización. Frecuentemente las aproximaciones para estudiar esta relación se orientan a medir la "satisfacción del usuario" que generalmente recopila información sobre las características favorables -o desfavorables- de la tecnología en uso -como interfaz de usuario, desempeño, facilidad de uso- (Doll \& Torkzadeh, 1988; Lucas, Ginzberg \& Schultz, 1990; Melone, 1990) y características generales sobre actitudes (Lucas, 1975; Ives, Olson and Baroudi, 1983). La equivalencia de la "satisfacción del usuario" con la "aceptación tecnológica" de Saga y Zmud (1994), por ejemplo, es un constructo teórico que carece de solidez (Melone, 1990).

La propuesta de evaluar la incorporación tecnológica justo desde lo que no contempla el CMM, representa -además de una contribución complementaria- otra manera de abordar la evaluación de la penetración tecnológica en la organización universitaria: a mayor nivel de relación humano-tecnológica, mayor incrustación de la implementación tecnológica, de manera profunda y comprensiva, en los sistemas de trabajo de una organización o individuo (Sullivan, 1985; Kwon, 1987; Cooper \& Zmud, 1990). Es decir, el nivel de relación humana-tecnológica como indicador de la integración tecnológica tiene sentido porque se entiende que en la medida en que la relación de los individuos con la tecnología implantada crece, en esa medida se cuenta con un sistema de información que se identifica con las tareas cotidianas, estratégicas y de toma de decisiones en la organización. La consistencia del marco teórico de Saga y Zmud (1994) y la apropiación tecnológica con la propuesta metodológica de la UNESCO (2009) resulta un hallazgo importante para el cimiento de la metodología que propone este 
trabajo de investigación: se operacionaliza mediante una metodología consensuada y probada a nivel internacional, lo que puede presagiar la viabilidad operativa.

La incorporación de las TIC en la institución universitaria frecuentemente se asocia con efectos visibles como el mejoramiento en el "ranking" de la calidad educativa o con la productividad científica o la capacidad profesional de sus egresados. El mejoramiento en estos aspectos depende en principio de las capacidades de la universidad. Las TIC son un instrumento que puede potenciar estas capacidades mediante su uso efectivo (Kozma, 2005; OECD, 2008; DEGV, 2011). En la aplicación preliminar de la metodología propuesta se observó que la evaluación del mejoramiento de las capacidades de la institución universitaria asociadas con las TIC debe fundamentarse inicialmente en la visión y voluntad para beneficiarse de la tecnología y si existen las condiciones para ello y en el uso efectivo y profundo en las funciones sustantivas universitarias, en este caso en la enseñanza aprendizaje.

Presentación del artículo: 26 de noviembre de 2015

Fecha de aprobación: 8 de enero de 2016

Fecha de publicación: 30 de enero de 2016

Nolasco-Vázquez, P. y Ojeda-Ramírez, M. (2016). La evaluación de la integración de las TIC en la educación superior: fundamento para una metodología. RED. Revista de Educación a Distancia. 48(9). Consultado el (dd/mm/aaaa) en http://www.um.es/ead/red/48/nolasco.pdf

\section{Bibliografía}

Álvarez, T. (2015). Diferencias en la apropiación tecnológica de los estudiantes de la Universidad Veracruzana: las licenciaturas de Biología, Ingeniería Civil, Historia y Derecho. Tesis de Maestría en Investigación Educativa. Instituto de Investigaciones en Educación. Universidad Veracruzana. Xalapa, Veracruz. Enero de 2015.

Australian and New Zealand Institute for Information Literacy. (2004). Australian and New Zealand Information Literacy Framework. Principles, Standards and Practice. Second Edition. Library Publications. University of South Australia. Recuperado de http://www.library.unisa.edu.au/Learn/infolit/infolit-2nd-edition.pdf (accesado el 20 de noviembre de 2013)

Association of College and Research Libraries (2000). Information Literacy Competency Standards for Higher Education. American Library Association. Chicago, Illinois. EUA. Recuperado de http://www.ala.org/acrl/sites/ala.org.acrl/files/content/standards/standards.pdf (accesado el 12 de noviembre de 2013).

Clark, R. (1983). El sistema de educación superior. Una visión comparativa de la organización académica. Universidad Autónoma Metropolitana-Azcapotzalco. México. D.F: Editorial Patria, S.A. de C.V., Primera Edición. 
Clark, R. (1986). Diversification of Higher Education viability and Change. The mockers and Mocked: Comparative Perspectives on Differentiation, Convergence and Diversity in Higher Education. Oxford: AUI Press, Pergamon.

Cobo, C. (2008). Aprendizaje adaptable y apropiación tecnológica: Reflexiones prospectivas. México: FLACSO México.

Colás, P., Rodríguez, M. \& Jiménez, R. (2005). Evaluación de E-learning. Indicadores de calidad desde el enfoque sociocultural. Teoría de La Educación: Educación y Cultura en La Sociedad de La Información. Recuperado de http://campus.usal.es/ teoriaeducacion/rev_numero_06_2/n6_02_art_colas_rodrigue z_jimenez.htm (accesado el 14 de noviembre de 2014)

Conferencia Ministerial de los Países de la Unión Europea, de América Latina y del Caribe sobre la Enseñanza Superior (2000). Declaración de la Conferencia Ministerial de los Países de la Unión Europea, de América Latina y del Caribe sobre la Enseñanza Superior. París, Francia, pp. 1-4. Recuperado de http://www.educacion.gob.es/dctm/mepsyd/educacion/universidades/politicainternacional/espaciocomun/6.declaracionparisesp.pdf?documentId=0901e72b8004874e (accesado el 22 de octubre de 2013).

Cooper, R., Zmud, R (1990). Information Technology Implementation Research: A technological diffusion approach. Management Science, 36, 2, 123-139

Council of Australian University Librarians (2001). Information Literacy Standards. First Edition. Acton: Library Publications. University of South Australia.

Crovi, D. (2009). Acceso, uso y apropiación de las TIC en comunidades académicas: Diagnóstico en la UNAM. México: Plaza y Valdez Editores..

Curtis, B., Hefley, W. \& Miller, S. (1995). Overview of the People Capability Maturity Model. Software Engineering Institute, Carnegie Mellon University, Pittsburg, Pensylvania. Recuperado de http://www.sei.cmu.edu/reports/93tr024.pdf (accesado el 30 de noviembre de 2013).

Davenport, T., Short, J. (1990). The New Industrial Engineering: Information Technology and Business Process Redesign. Sloan Management Review. Summer, $11-27$

Davis, F. (1986). A Technology Acceptance Model for Empirically Testing New EndUser Information Systems: Theory and Results. Doctoral dissertation, Sloan School of Management, MIT.

Davis, F., (1989). Perceived Usefulness, Perceived Ease of Use, and End-User Acceptance of Information Technology. MIS Quarterly, 13, 318-339

Departamento de Educación del Gobierno Vasco, (2011). Modelo de madurez tecnológica de centro educativo. Gobierno del País Vasco. España. Recuperado de 
http://www.hezkuntza.ejgv.euskadi.net/r43-

573/es/contenidos/informacion/dig_publicaciones_innovacion/es_tecnolog/adjuntos/ 20_ikt_400/400004c_Pub_EJ_Madurez_TIC_c.pdf (accesado el 14 de octubre de 2013).

Dettmer, J. (2004). Globalización, Convergencia y Diferenciación de la Educación Superior: Una Revisión Teórico-Conceptual. Revista de la Educación Superior. Vol. XXXII(4). No. 132. Octubre-Diciembre de 2004. ISSN: 0185-2760. Recuperado de http://www.ses.unam.mx/curso2007/pdf/Dettmer.pdf (accesado el 10 de enero de 2015).

Doll, W., Torkzadeh, G. (1988). The Measurement of End-User Computing Satsifaction. MIS Quarterly. June, 259-274

Duarte, D. \& Ventura, P. (2011). Towards a Maturity Model for Higher Education Institutions. SAPIENTIA, Repositório Institucional Universidade do Algarve. Recuperado de http://ceur-ws.org/Vol-731/05.pdf

Fernández, K. (2015). Apropiación tecnológica de estudiantes: Estudios de casos por modalidad educativa en universidades de México, Guatemala y Venezuela. Tesis doctoral por publicar. Instituto de Investigaciones en Educación. Universidad Veracruzana. Xalapa, Veracruz. Marzo de 2015.

Fernández, A. \& Llorens, F. (2011). Gobierno de las TIC para universidades. Conferencia de Rectores de las Universidades Españolas (CRUE). Madrid, España. Recuperado de http://www.crue.org/export/sites/Crue/Publicaciones/Documentos/GobiernoTI/gobier no_de_las_TI_para_universidades.pdf (accesado el 27 de octubre de 2013).

Hammer, M. (1990). Reengineering Work: Don't Automate, Obbliterate. Harvard Business Review, July, 104-112

INFOTEC (2014). El valor de la etnografía para el diseño de productos, servicios y políticas TIC. Recuperado de http://infotec.com.mx/work/models/infotec/Resource/6149/1/images/MS3_com_SE MINARIO.pdf\#page=95 (accesado el 15 de enero de 2015).

IT Governance Institute (2007). COBIT 4.1. Framework, Control Objectives, Management Guidelines, Maturity Models. IT Governance Institute, Rolling Meadows, IL, USA.

Ives, B., Olson, M., Baroudi, J. (1983). The Measurement of User Information Satisfaction. CACM, 26, 10, 785-793

King, R. (1991). The Transformation of Organizational Information Systms: Beyond the Implementation stage. Procedings of the American Chinese Management Association. August. 
Kling, R., Lacono, S. (1984). The Control of Information Systems Developments After Implementation. Communication of the ACM. December, 27, 1218-1226

Kozma, R. (2005). National policies that connect ict-based education reform to economic and social development. Human Technology. Volume 1 (2), pp. 117-156. Recuperado de https://jyx.jyu.fi/dspace/bitstream/handle/123456789/20179/HT_2005_v01_n02_p_1 17-156.pdf?sequence $=1$ (accesado el 14 de octubre de 2013)

Kwon, T., Zmud, R. (2005). Unifyng the Fragmented Models of Informations Systmes Implementation. In R. Booland and R. Hirscheim (Eds.). Critical Issues in Information Systems Research. Nueva York, John Wiley and Sons. 227-251.

Kwon, T., (1987). A Study of The Influence of Communications Networks on MIS Institutionalization. Dissertation.

López, Z. (2015). Propuesta de un marco de referencia para el apropiamiento tecnológico en instituciones de Educación Superior. Caso de estudio: Universidad Veracruzana. Tesis para obtener el Grado de Maestra en Computación Aplicada. Laboratorio Nacional de Informática Avanzada. Centro de Enseñanza LANIA. Xalapa, Veracruz, México. Febrero de 2015.

Lucas, H. (1975). Performance and the Use of an Information System. Management Science, 21, 8, 908-919

Lucas, H., Ginzberg, M., Schultz, R. (1990). Information Systems Implementation: Testing a Structural Model. New Jersey: Ablex.

Melone, N. (1990). A Theoretical Assessment of the User-Satisfaction Construct in Information Systems Research. Management Science, 36, 1, January, 76-91

Neave, G. (1996). Homogenization, integration and convergence: The cheshire cats of higher education analysis. The mockers and mocked: comparative perspectives on differentiation, convergence and diversity in higher education. Acton: AUI Press, Pergamon.

Organización de Estados Iberoamericanos, (1999). Declaración de Río de Janeiro. Primera Cumbre entre los Jefes de Estado y de Gobierno de América Latina y el Caribe y la Unión Europea. Río de Janeiro, Brasil. Recuperado de http://www.oei.es/cumbrerio.htm (accesado el 4 de noviembre de 2013)

Organización de las Naciones Unidas para la Educación, la Ciencia y la Cultura (1998). Conferencia Mundial sobre la Educación Superior. La Educación Superior en el Siglo XXI. Siglo XXI: Visión y Acción. Tomo I. Informe final. París, Francia. Recuperado de http://unesdoc.unesco.org/images/0011/001163/116345s.pdf (accesado el 2 de noviembre de 2013)

Organización de las Naciones Unidas para la Educación, la Ciencia y la Cultura (2009). Medición de las Tecnologías de la Información y la Comunicación (TIC) en 
Educación - Manual del Usuario. Documento Técnico No. 2. Instituto de Estadística de la UNESCO. Montreal, Québec, Canada. Recuperado de http://www.uis.unesco.org/Education/Documents/isced-2011-sp.pdf (accesado el 10 de agosto de 2014)

Organización de las Naciones Unidas para la Educación, la Ciencia y la Cultura (2011). Clasificación Internacional Normalizada de la Educación CINE 2011. Instituto de Estadística de la UNESCO. Montreal, Québec, Canada. Recuperado de http://www.uis.unesco.org/Communication/Documents/ict-regional-survey-lac-2012sp.pdf (accesado el 22 de septiembre de 2013)

Organización de las Naciones Unidas para la Educación, la Ciencia y la Cultura (2013). Uso de TIC en educación en América Latina y el Caribe. Análisis regional de la integración de las TIC en la educación y de la aptitud digital (e-readiness). Instituto de Estadística de la UNESCO. Montreal, Québec, Canada. Recuperado de http://www.uis.unesco.org/Communication/Documents/ict-regional-survey-lac-2012sp.pdf (accesado el 22 de septiembre de 2013)

Organization for Economic Co-operation and Development (2008). Tertiaty Education for the Knowledge Society. OECD Thematic Review of Tertiery Education: Synthesis Report. Overview. OECD.

Overdijk, M., \& van Diggelen, W. (2006). Technology Appropriation in Face-to-Face Collaborative Learning. First European Conference on Technology Enhanced Learning. Recuperado de http://ceur-ws.org/Vol-213/paper17.pdf (Accesado en noviembre de 2014)

Paulk, M., Curtis, B., Chrissis, M. \& Webber, C. (1993). Capability Maturity Model for Software, Versión 1.1. Technical Report. Software Engineering Institute, Carnegie Mellon University, Pittsburg, Pensylvania. Recuperado de http://resources.sei.cmu.edu/asset_files/TechnicalReport/1993_005_001_16211.pdf (accesado el 10 de enero de 2015).

Peng, S. \& Wang, L. (2008). Pursuing quality and equity of higher education: A review of policies and practices in Easy Asia. Wiley InterScience. Vol. 2008, pp. 25-42. Recuperado de http://onlinelibrary.wiley.com/doi/10.1002/ir.276/pdf (accesado en 20 de noviembre de 2012).

Pimienta, D. (2007). Brecha digital, brecha social, brecha paradigmática. Funredes. Recuperado de http://ictlogy.net/bibliography/reports/projects.php?idp=1286 Accesado en septiembre de 2014

Quijano, A. (2007). Aceptación de tecnologías de información y cambio organizacional: propuesta metodológica para su planeación en una biblioteca académica. Tesis doctoral. Universidad Nacional Autónoma de México. México, D.F. 
Ritti, R., Silver, J. (1986). Early Processes of Institutionalization: The Dramaturgy of Exchange in Interorganizationl relations. Administrative Sicence Quarterly, 31, 2542

Secretaría de Comunicaciones y Transportes (2012). Agenda Digital.mx. 1ra. Edición. ISBN: 978-607-95879-0-1. México. Recuperado de http://www.agendadigital.mx/descargas/AgendaDigitalmx.pdf

Sheppard, B., Hartwick, H., Warshaw, P., (1988). The Theory of Reasoned Action: A Meta-Analysis of Past Research with Recommendations for Modifications and Future Research. Journal of Consumer Research, 15, 325-343

Snider, J., Osgood, C. (1969). Semantic Differential Technique. Chicago: Aldane.

Saga, V.L. y R.W. Zmud (1994). "The Nature and Determinations of It-Acceptance, Routinization and Infusion", en L.Levine (ed), Difusion, Transfer and Implementation of Information Technology, Elsevier Science B.V. (North-Holland), pp. 67-86

Sullivan, C. (1985). Systems Planning in the Information Age”. Sloan Management Review, 26-2, 3-11

Unión Internacional de Telecomunicaciones (2010). Indicadores Clave sobre TIC. Partnership para la Medición de las TIC para el Desarrollo. Ginebra, Suiza. Recuperado de http://www.itu.int/dms_pub/itu-d/opb/ind/D-IND-ICT_CORE-2010PDF-S.pdf (accesado el 25 de noviembre de 2013)

Unión Internacional de Telecomunicaciones (2014). Informe sobre Medición de la Sociedad de la Información 2014. Resumen Ejecutivo. Ginebra, Suiza. Recuperado de http://www.itu.int/en/ITU-

D/Statistics/Documents/publications/mis2014/MIS_2014_Exec-sum-S.pdf (accesado el 13 de febrero de 2015)

Universidad Veracruzana (2013). Programa de Trabajo Estratégico 2013 - 2017. Universidad Veracruzana. Xalapa, Veracruz. Recuperado de http://www.uv.mx/programa-trabajo/Programa-de-Trabajo-Estrategico-version-parapantalla.pdf (acceso 13-10-2013).

Van Dijk, J. (2005). The Deepening Divide: Inequality in the Information Society. California: SAGE.

Vught, F. (1986). Isomorphism in higher education? Towards a theory of differentiation and diversity in higher education systems., The mockers and mocked: comparative perspectives on differentiation, convergence and diversity in higher education. Lynn Meek, Leo Goedegebuure, Osmo Kivinen y Risto Rinne. AUI Press, Pergamon.

World Economic Forum (2015). The Global Information Technology Report 2015. ICTs for Inclusive Growth. Insight Report. Soumitra Dutta, Thierry Geiger, Bruno Lanvin, 
Editores. INSEAD, The Bussiness School of the World. Recuperado de http://reports.weforum.org/global-information-technology-report-2015/

World Wide Web Foundation, (2012). Web Index 2012. Recuperado de http://thewebindex.org/2012/10/2012-Web-Index-Key-Findings.pdf (accesado el 16 de octubre de 2012)

World Wide Web Foundation, (2013). Web Index Report 2013. Recuperado de http://thewebindex.org/wp-content/uploads/2013/11/Web-Index-Annual-Report2013-FINAL.pdf (accesado el 29 de julio de 2014)

World Wide Web Foundation, (2014). Web Index Report 2014-15. Recuperado de http://thewebindex.org/wpcontent/uploads/2014/12/Web_Index_24pp_November2014.pdf (accesado el 12 de junio de 2015)

Yin, R. (1979). Changing Urban Bureaucracies. Santa Mónica: Lexington Books.

Zmud, R., Apple, L. (1992). Measuring Technology Incorporation/Infusion. Journal of Product Innovation Management

Zucker, L. (1977). The Role of Institutionalization in Cultural Persistence". American Sociological Review, 42, 5, 726-743

Zubieta, J., Bautista, T., Quijano, A. (2011). Aceptación de las TIC en la docencia: una tipología de los académicos de la UNAM. México, D.F.: Universidad Nacional Autónoma de México. 